Virginia Commonwealth University vCU Scholars Compass

2005

\title{
Thermodynamics of folding and association of lattice-model proteins
}

Troy Cellmer

University of California, Berkeley

Dusan Bratko

Virginia Commonwealth University, University of California, Berkeley

John M. Prausnitz

University of California, Berkeley

Harvey Blanch

University of California, Berkeley

Follow this and additional works at: http://scholarscompass.vcu.edu/chem_pubs

Part of the Chemistry Commons

Cellmer, T., Bratko, D., Prausnitz, J. M., et al. Thermodynamics of folding and association of lattice-model proteins. The Journal of Chemical Physics 122, 174908 (2005). Copyright (C) 2005 AIP Publishing LLC.

\section{Downloaded from}

http://scholarscompass.vcu.edu/chem_pubs/58

This Article is brought to you for free and open access by the Dept. of Chemistry at VCU Scholars Compass. It has been accepted for inclusion in Chemistry Publications by an authorized administrator of VCU Scholars Compass. For more information, please contact libcompass@vcu.edu. 


\title{
Thermodynamics of folding and association of lattice-model proteins
}

\author{
Troy Cellmer \\ Department of Chemical Engineering, University of California, Berkeley, California 94720 \\ Dusan Bratko \\ Department of Chemistry, Virginia Commonwealth University, Richmond, Virginia 23284 \\ and Department of Chemical Engineering, University of California, Berkeley, California 94720 \\ John M. Prausnitz \\ Department of Chemical Engineering, University of California, Berkeley, California 94720 \\ and Chemical Sciences Division, Lawrence Berkeley National Laboratory, Berkeley, California 94720 \\ Harvey Blanch ${ }^{\text {a) }}$ \\ Department of Chemical Engineering, University of California, Berkeley, California 94720
}

(Received 26 January 2005; accepted 17 February 2005; published online 4 May 2005)

\begin{abstract}
Closely related to the "protein folding problem" is the issue of protein misfolding and aggregation. Protein aggregation has been associated with the pathologies of nearly 20 human diseases and presents serious difficulties during the manufacture of pharmaceutical proteins. Computational studies of multiprotein systems have recently emerged as a powerful complement to experimental efforts aimed at understanding the mechanisms of protein aggregation. We describe the thermodynamics of systems containing two lattice-model 64-mers. A parallel tempering algorithm abates problems associated with glassy systems and the weighted histogram analysis method improves statistical quality. The presence of a second chain has a substantial effect on single-chain conformational preferences. The melting temperature is substantially reduced, and the increase in the population of unfolded states is correlated with an increase in interactions between chains. The transition from two native chains to a non-native aggregate is entropically favorable. Non-native aggregates receive $\sim 25 \%$ of their stabilizing energy from intraprotein contacts not found in the lowest-energy structure. Contact maps show that for non-native dimers, nearly $50 \%$ of the most probable interprotein contacts involve pairs of residues that form native contacts, suggesting that a domain-swapping mechanism is involved in self-association. (c) 2005 American Institute of Physics.

[DOI: $10.1063 / 1.1888545]$
\end{abstract}

\section{INTRODUCTION}

The native state of a protein is its most stable conformation and is intimately linked with its biological function. How proteins resolve the Levinthal paradox and attain their native state has been the subject of intense experimental and theoretical study for more than 30 years. An apparent solution to the paradox has been obtained via the generation of free-energy surfaces or landscapes, which can be determined for simplified model proteins via computer simulation. ${ }^{1-3}$ In short, the shape of energy landscapes suggests that folding is a stochastic process where the search for the native state corresponds to a gradual descent toward the global energy minimum. The energetic bias toward the native state ensures that a folding protein need not search through an impossibly large number of configurations to fold properly.

While such studies have been helpful in determining mechanisms of protein folding in isolation, in many systems of practical interest proteins must fold in a crowded environment, an effect sure to perturb folding landscapes. One such system is in vivo folding, where proteins interact with other biomolecules after synthesis. Proteins can misfold and form

\footnotetext{
a) Author to whom correspondence should be addressed. Electronic mail: blanch@berkeley.edu
}

aggregates believed to be pathological. Such aggregates are suspected to cause Alzheimer's, Parkinson's, and Huntington's diseases, as well as more than another dozen human diseases. ${ }^{1,4}$ Further, protein aggregation can present serious challenges during the manufacture and storage of pharmaceutical proteins. ${ }^{5-8}$

As a logical extension of computational work aimed at determining mechanisms of protein folding in isolation, simulations have recently emerged as a powerful tool to investigate protein misfolding and aggregation. Protein representations have ranged from two- and three-dimensional lattice models ${ }^{9-15}$ to more realistic off-lattice models capable of mimicking the folding properties of real proteins. ${ }^{16}$ To minimize the number of simulated particles, solvent is normally included implicitly in the potential function (dependence on the primary chain conformation renders analogous integrating-out of the effect of adjacent chains impractical). While the simplicity of coarse-grained models limits direct comparisons to specific experimental systems, such models offer opportunities to make generalizations to guide experimental research. Simulations of multichain systems have been used to investigate protein aggregation in multiple contexts, including protein refolding, ${ }^{17,18}$ prion propagation, ${ }^{19,20}$ and amyloid fibril formation. ${ }^{16}$ 
This report presents results pertaining to the thermodynamics of systems containing either one or two lattice-model 64-mers. To extend studies of protein folding in isolation, our primary aim has been to study the effects of interprotein interactions on single-chain conformational preferences. A second aim was to ascertain the thermodynamic driving forces that establish the partitioning between monomeric and aggregated states. A more complete understanding of these driving forces will facilitate efforts focused on uncovering the factors that determine a protein's tendency to selfassociate. Knowledge of these factors can then be exploited to rationally design mutations aimed at reducing aggregation propensity.

To ease computational restraints associated with relatively large systems and to ensure reliable statistics, proteins are simulated as lattice model hetero-polymers. Each chain consists of 64 beads, a substantially larger chain size than lattice models employed in most published multichain studies. A modified 20-letter Miyazawa-Jernigan (MJ) ${ }^{21}$ potential function describes interactions between beads. ${ }^{22}$ While the MJ potential is empirical, the forces that govern aminoacid interactions are much too complex to be correctly represented by a simple pair potential function. Thus, while direct comparisons to experimental data are not possible, it is reasonable to assume that the MJ potential more correctly reflects the multitude of strong and weak forces that govern protein interactions than other more simple potentials commonly used for lattice models. ${ }^{23}$ A parallel tempering algorithm circumvents difficulties associated with simulating systems that often exhibit glass-like behavior. Last, the weighted histogram analysis method (WHAM) ${ }^{24}$ provides the best possible data quality from the given number of simulations.

A brief outline of the manuscript is as follows. First, the thermodynamics of single-chain folding is characterized, and the native state is defined. A calorimetric cooperativity for the model is then calculated, and compared to experimental data. Results from single-chain systems are then compared to systems containing pairs of chains. The effects of neighboring chains on single-molecule conformational preferences are analyzed using heat-capacity data, thermodynamic averages of several conformational metrics, and free-energy surfaces generated as a function of intramolecular and intermolecular contacts. We then present contact maps that provide insight into the specific amino acids involved in selfassociation.

\section{METHODS}

The solution was modeled using the conventional onlattice representation. Protein molecules are represented as self-avoiding chains comprised of amino-acid residues (or beads) interacting through renormalized $\mathrm{MJ}^{21}$ potentials. These potentials were determined by a statistical analysis of protein structures collected in the Brookhaven Protein Data Bank and can be accurately represented by a reduced set of only 23 parameters obtained through eigenvalue decomposition of the original interaction matrix. ${ }^{25}$ Interactions are assumed to be isotropic, short-ranged, and pairwise additive.
The short-ranged character of bead-bead interactions limits such interactions to pairs occupying neighboring lattice sites. To improve the folding behavior and aggregation resistance of the model proteins, Leonhard et al. ${ }^{26}$ proposed a twoparameter normalization of the original MJ potentials. Their procedure adjusts the residue selectivities and uniformly increases the nonspecific (homopolymeric) term in the beadbead attraction. This proposed modification of pair potentials between residues $i$ and $j$ was originally formulated within the context of the Ising model with explicit solute-solute $(i j)$, solute-solvent (iO), and solvent-solvent (00) interactions. ${ }^{22,26,27}$ Using the notation from the original work of Miyazawa and Jernigan, ${ }^{21}$ the solvent-averaged (implicit) interbead potentials, $e_{i j}$, can be related to explicit pair interactions $E_{i j}$ by

$$
e_{i j}=E_{i j}-E_{i 0}-E_{j 0}+E_{00} .
$$

While using a somewhat different formalism, Leonhard et $a .^{22}$ propose to adjust the residue-water interactions $\left(E_{i 0}\right)$ using a two-parameter function. Using notation consistent with Eq. (1), the adjusted residue-water interaction, $\widetilde{E_{i 0}}$ can be written as follows:

$$
\tilde{E}_{i 0}=E_{i 0}+\frac{1}{2}\left(1-C_{s}\right) e_{i i}+\varpi+\frac{C_{s}}{2 n} \sum_{i=1}^{n} e_{i i} .
$$

Here, $C_{s}$ and $\varpi$ represent the two adjustable parameters of the model. The summation over $i$ is performed over all amino-acid residue types; hence, $n=20$. Setting $C_{s}=1$ and $\varpi=-\left(C_{s} / 2 n\right) \sum_{i=1}^{n} e_{i i}$, recovers the original water-residue potentials. Using $C_{s}<1$ results in reduced hydrophobicity/ hydrophilicity-based discrimination between the residues. Deviations in $\varpi$ from $\left(C_{s} / 2 n\right) \sum_{i=1}^{n} e_{i i}$ lead to a uniform shift of rescaled solvent-residue potentials for all residues. In view of Eq. (1), both effects modulate the effective interresidue potentials. A detailed discussion of the above-noted procedure is available in Refs. 22,26,27.

In contrast to these works, here we employ an implicit solvent representation, with the solvent treated as a uniform background whose presence is reflected in the effective (MacMillan-Mayer) interresidue potentials $\nu_{i j}$. Here, $\nu_{i j}$ denotes the renormalized inter-residue potentials that will, in general, differ from the original implicit interactions, $e_{i j}$, reported in Table V of Miyazawa and Jernigan. ${ }^{21}$ For a selfavoiding chain with the range of interactions equal to the lattice spacing $a$, combining Eqs. (1) and (2) gives

$$
\begin{aligned}
\nu_{i j}= & E_{i j}-\widetilde{E}_{i 0}-\widetilde{E}_{j 0}+E_{00} \\
= & e_{i j}-\frac{1}{2}\left(1-C_{s}\right)\left(e_{i i}+e_{j j}\right)-2 \varpi \\
& -\frac{C_{s}}{n} \sum_{i=1}^{n} e_{i i} \quad \text { if }\left|\left(r_{i, m}-r_{j, m}\right)\right|=a,
\end{aligned}
$$

while $\nu_{i j}\left(r_{i, m}-r_{j, m}\right)=0 \quad$ if $\left|\left(r_{i, m}-r_{j, m}\right)\right|>a \quad$ or $|i-j|=1$ and $\nu_{i j}\left(r_{i, m}-r_{j, m}\right)=\infty$ if $\left|\left(r_{i, m}-r_{j, m}\right)\right|=0$,

We use the 64-mer sequence KEKSTAGRVASGVLDSVACG- 


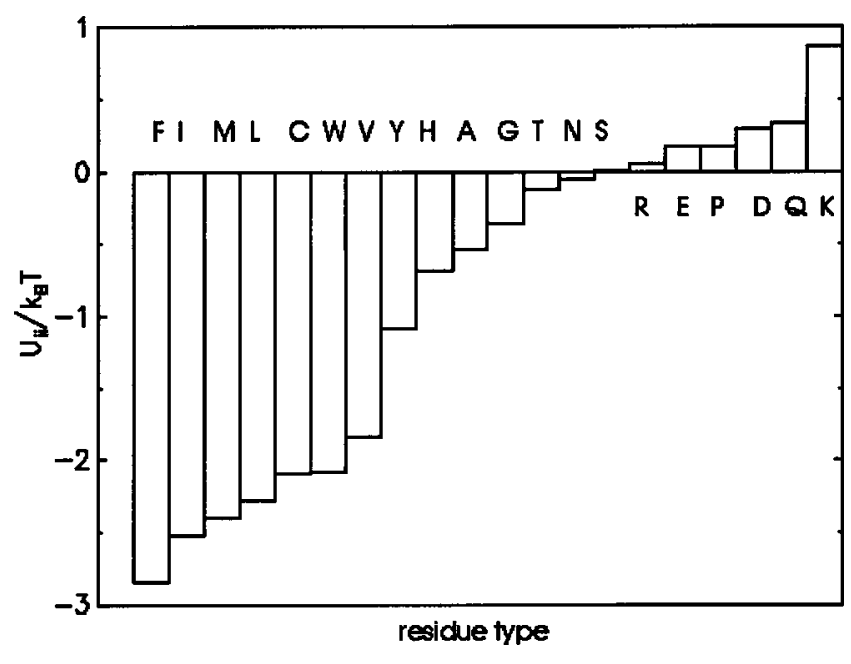

FIG. 1. Contact values of implicit (solvent-averaged) inter-residue potentials $u_{i i}\left(r_{i i}=a\right)$ at $T=0.858 T_{m}$, and at $C_{s}=0.2$ and $\varpi=0.16$.

VLGDIDTLQGSPIAKLKTFYGNKFNDVEASQAHMIRWP NY$T L P E$. We set our two adjustable parameters, $C_{s}=0.2$ and $\varpi=0.16$ because they optimize ${ }^{22,26}$ the protein-like behavior of the selected model polypeptide. Our lowest temperature $T=T_{0}$, is set to 0.375 , which corresponds to $0.858 T_{m}$, where $T_{m}$ is the melting (thermal unfolding) temperature of the isolated protein. To illustrate relative residue hydrophobicities (hydrophilicities) in the rescaled model, Fig. 1 compares the contact values of implicit (solvent-averaged) potentials $\nu_{i i}\left(r_{i i}=a\right)$ for all 20 amino-acid residues at $T_{0}$. The corresponding melting temperature obtained from our simulations (see the following), $T_{m}=0.437$, is in excellent agreement with $T_{m}=0.43$ reported in Ref. 26 using the explicit-solvent, Ising-type Hamiltonian. In analogy with our previous work, ${ }^{9,10}$ we express the energy function of $M$ interacting chains, each containing $N$ monomer units in terms of implicit inter-residue potentials:

$$
\begin{aligned}
V(\{r\})= & \sum_{m=1}^{M} \sum_{i=1}^{N-2} \sum_{j=i+1}^{N} \nu_{i j}\left(r_{i, m}-r_{j, m}\right) \\
& +\sum_{m=1}^{M-1} \sum_{n=m+1}^{M} \sum_{i=1}^{N} \sum_{j=1}^{N} \nu_{i j}\left(r_{i, m}-r_{j, n}\right) .
\end{aligned}
$$

Lattice step $a$ is equal to the monomer length, and $\mathbf{r}_{i, m}$ is the position of the $i$ th bead of chain $m$; hence, the $3(M N-1)$ dimensional vector ${ }^{9}$ contains a complete description of the (translationally invariant) system.

Folding and association behavior of the chains is studied by canonical $(N, V, T)$ Monte Carlo simulations of up to two freely moving protein molecules placed in the cubic simulation box of size L. Protein concentration is defined by volume fraction $f_{V}=N M / L^{3}$. Boundary effects are taken into account by applying periodic (minimum image) conditions ${ }^{28}$ in all three directions. We use standard simulation moves ${ }^{29}$ including; (1) displacements of either one of the end beads to one of the available four neighboring sites; (2) corner flips for beads characterized by a right angle between the directions to both contour neighbors; (3) crankshaft moves of bead pairs located at the bottom of a $\mathrm{U}$ turn. We also allow forward and backward slithering-snake reptation moves, ${ }^{30}$ as well as translations of entire chains. Moves of different types are attempted at random with a priori probabilities specified in Ref. 9 where further details of the $(N, V, T)$ simulations are available.

To alleviate problems related to local trapping on the rugged free energy landscape of aggregating proteins, we apply a nondynamic replica exchange Monte Carlo simulation technique (REMC). ${ }^{10}$ This technique facilitates barrier crossings by simultaneously sampling several replicas of a given model system, each replica held at a slightly different temperature. In addition to standard simulation moves (vide supra), the systems are allowed to swap between adjacent temperature levels with probabilities that preserve canonical (Boltzmann) statistics within each level. Assuming an approximate Arrhenius dependence of first passage times from the local minimum, the chance of escape of a trapped system is significantly improved during the time it spends at an elevated temperature. A temperature swap was attempted after every simulation pass, i.e., a sequence of attempted moves of all the beads in the system. The attempted exchange of systems $i$ and $j$ between temperature levels $m$ and $n$, was accepted with the probability: ${ }^{31}$

$$
p_{s}=\min \left\{1, \exp \left[-\left(\frac{1}{k_{B} T_{n}}-\frac{1}{k_{B} T_{m}}\right)\left(V_{i}-V_{j}\right)\right]\right\} .
$$

For two-chain systems, the number of replicas (and temperature levels) was six, with temperature $T$ ranging between $T_{0}$ and $1.3 T_{0}$, and typical swapping acceptances between $8 \%$ and $30 \%$. The number of the replicas and temperature spacing were determined empirically in our earlier work. ${ }^{10}$ Similar simulation conditions were selected in a more recent study of a continuum model of peptide aggregation and produced about equal acceptances of attempted replica exchanges. ${ }^{32}$ For simulations of isolated chains, data from three different parallel tempering trajectories were used for a total of 18 simulations. Temperatures from this data set ranged from $0.3 T_{0}$ to $3.0 T_{0}$. In addition to improved configuration dynamics, the REMC technique simultaneously provided samples of characteristic model variables at a set of different temperatures.

WHAM analysis. WHAM ${ }^{24}$ was used to analyze simulation data. WHAM minimizes the error in the density-ofstates function, and facilitates the calculation of free-energy surfaces. In each simulation, six quantities were monitored: the total system potential energy $V$; the total number of native contacts $N_{\text {Nat }}$; the total number of interprotein contacts $N_{\text {inter }}$; the contribution to the overall potential from interactions between beads on the same chain $V_{\text {intra }}$; the contribution to the overall potential from interactions between beads on different chains $V_{\text {inter }}$; and the average radius of gyration $R_{g}$.

Calculating the density-of-states function $(\Omega)$ for six quantities is computationally impractical. Thus it was necessary to calculate several $\Omega$, each a function of different thermodynamic parameters. A generic description of $\Omega$ is 


$$
\Omega\left(V, \xi_{1}, \xi_{2}\right)=\frac{\sum_{j=1}^{k} N_{k}\left(V, \xi_{1}, \xi_{2}\right)}{\sum_{j=1}^{k} n_{j} \exp \left(-f_{j}-\beta_{j} V\right)},
$$

where $\xi_{1}$ and $\xi_{2}$ are any of the above-mentioned parameters, $N_{k}$ is the number of occurrences for samples with $\left(V, \xi_{1}, \xi_{2}\right), f_{j}$ is equal to $\beta A_{\mathrm{j}}$ where $A_{j}$ is the free energy for simulation $j, \beta$ is $1 / k_{B} T, k$ is the number of simulations, and $n_{j}$ is the number of samples from simulation $j$. Free energies were calculated by solving

$$
\begin{aligned}
& P_{\beta}\left(V, \xi_{1}, \xi_{2}\right)=\frac{\sum_{i=1}^{k} N_{i}\left(V, \xi_{1}, \xi_{2}\right) \exp (-\beta V)}{\sum_{j=1}^{k} n_{j} \exp \left(-f_{j}-\beta_{j} V\right)}, \\
& \exp \left(-f_{k}\right)=\sum_{V, \xi_{1}, \xi_{2}} P_{\beta_{k}}\left(V, \xi_{1}, \xi_{2}\right),
\end{aligned}
$$

where $P_{\beta}$ is the probability of observing a sample with $\left(V, \xi_{1}, \xi_{2}\right)$. Thermodynamic averages are then calculated from

$$
\left\langle\xi_{1}\right\rangle=\frac{\sum_{V, \xi_{1}, \xi_{2}}\left(\xi_{1}\right)^{*} \Omega\left(V, \xi_{1}, \xi_{2}\right) \exp (-\beta V)}{\sum_{V, \xi_{1}, \xi_{2}} \Omega\left(V, \xi_{1}, \xi_{2}\right) \exp (-\beta V)}
$$

using $\xi_{1}$ as an example. Free energies are calculated via

$$
\begin{aligned}
& F\left(\xi_{1}, \xi_{2}\right)=-k_{b} T \ln \left\{P_{\beta}\left(\xi_{1}, \xi_{2}\right)\right\}, \\
& P_{\beta}\left(\xi_{1}, \xi_{2}\right)=\sum_{V} P_{\beta}\left(V, \xi_{1}, \xi_{2}\right) .
\end{aligned}
$$

For simplicity, all energies are reported in units of $k_{B} T_{0}$ and temperature in units of $T_{0}$.

\section{RESULTS AND DISCUSSION}

\section{A. Characterization of isolated chains}

A snapshot of the lowest-energy structure (LES) ${ }^{26}$ is shown in Fig. 2, while Fig. 2(a) shows a contact map of the LES. A black box at the position $(i, j)$ in the contact map indicates that the beads $i, j$ form a contact in the lowest energy structure. All of the boxes are filled along the diagonal $i=j+1$, as these positions represent pairs of beads that are adjacent in primary sequence and therefore always bonded. The high population of contacts along the curve $i=j+3$ reflects the high contact probability for residues separated by the minimal contour distance that allows a contact between two beads that are not bonded. In contrast to these trivial contacts, special significance pertains to contacts between residue pairs that are separated by a considerable contour distance $|i-j|$. The farther away from the diagonal the black box is located, the larger the distance between the beads in the primary sequence. The plot will be revisited later, for comparison to contact maps generated under conditions where multiple chains are present.

The single-chain folding thermodynamics of the model sequence, and its corresponding potential function, have been studied previously. ${ }^{26,27,22}$ To help interpret results from
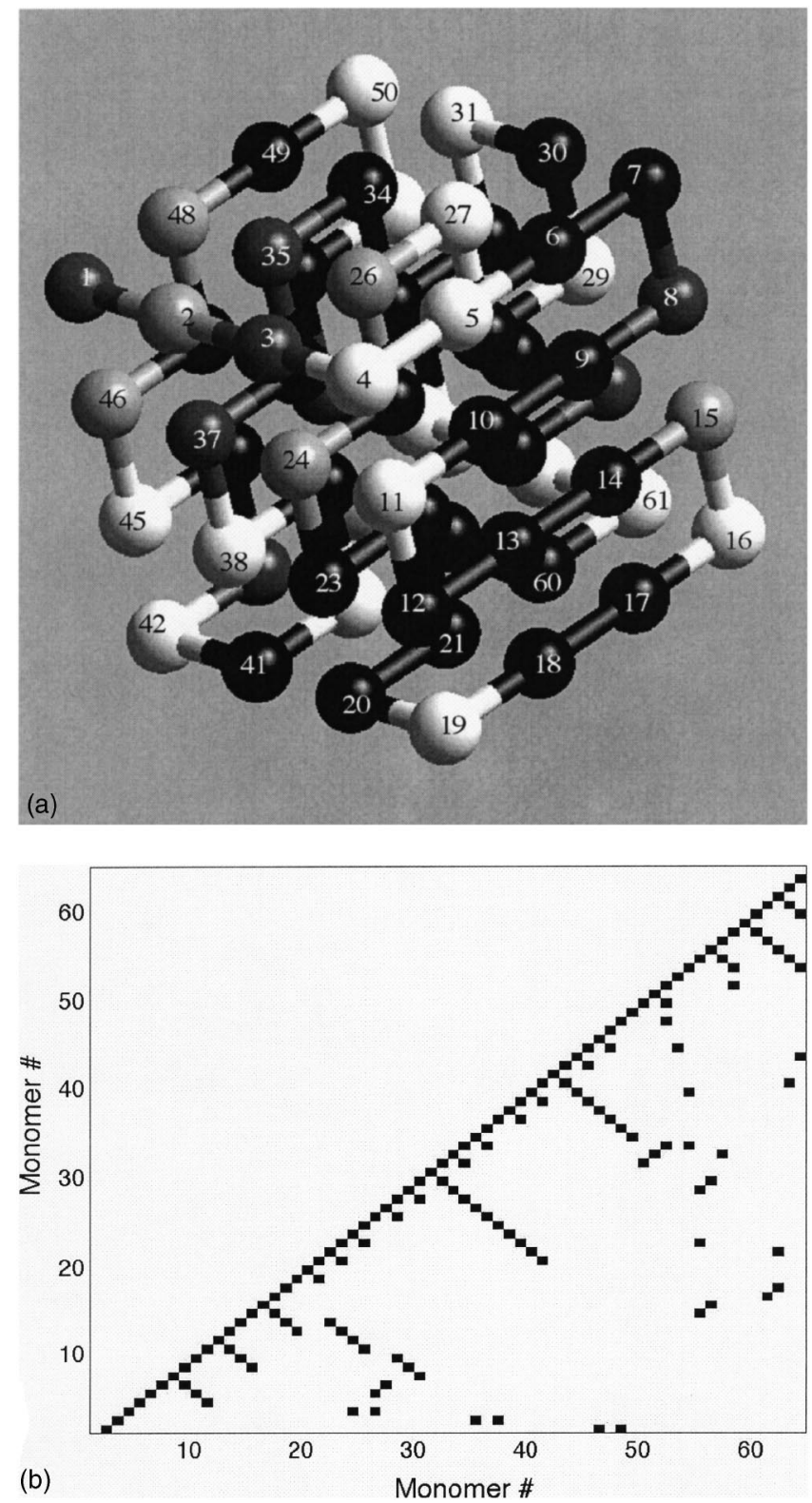

FIG. 2. (a) Lowest energy structure (LES) for the model 64-mer. Nonpolar residues are in black (GAVLIMPFW), polar residues in white (STNQYCK), negatively charged residues in light grey (DEH), and positively charged residues in dark grey (KR). (b). Contact map for the LES. A black box at the position $(i, j)$ indicates beads $i, j$ form a contact in the LES.

multichain simulations, a more rigorous thermodynamic description of an isolated chain was calculated. Figure 3(a) shows the heat capacity of a single chain as a function of temperature. The peak at $T=1.155$ indicates a weak firstorder phase transition; this peak corresponds to the folding temperature. Figure 3(b) shows the free energy as a function of potential energy $(V)$ at $T=1.155$; there are two distinct minima that have virtually equal free energies. The local maximum at $V=-75$ (corresponding to vanishing slope of the energy histogram) is the thermodynamic barrier to folding; therefore, we define configurations with $V<-75$ as native. The fraction folded $\left(F_{\text {fold }}\right)$ as a function of temperature [Fig. 2(c)] is 

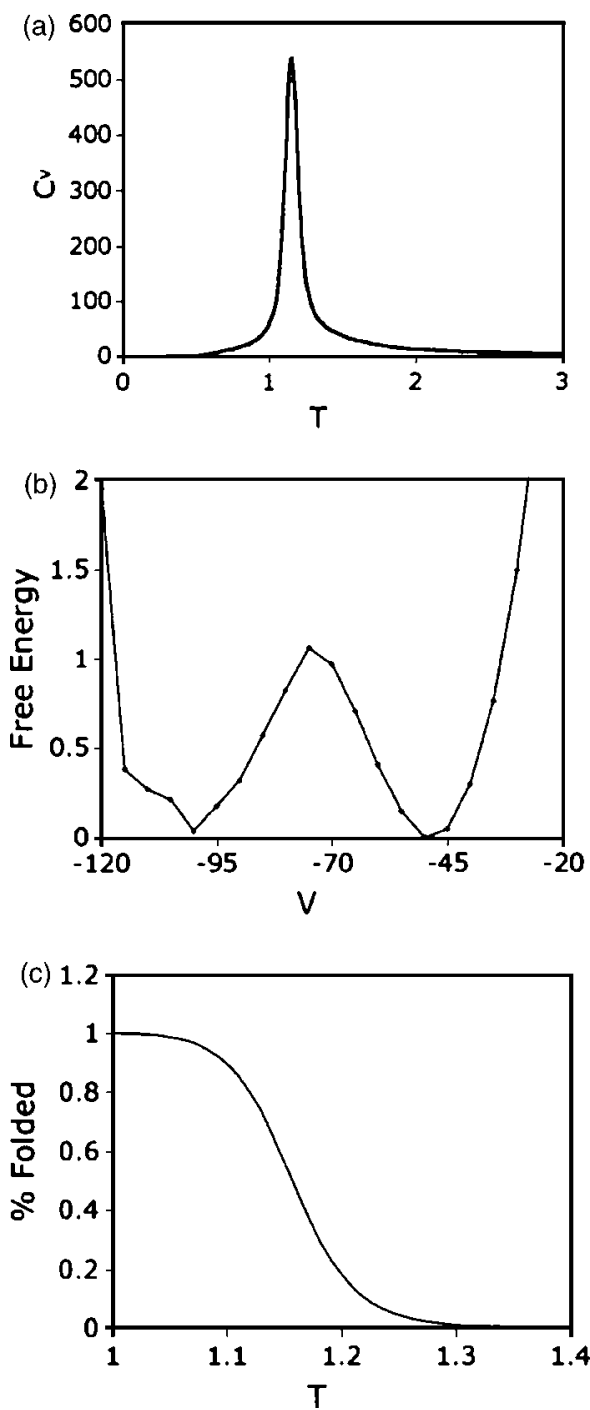

FIG. 3. (a) Heat capacity $\left(C_{v}\right)$ as a function of reduced temperature $(T)$. (b) Free energy as a function of potential energy $(\mathrm{V})$ at $T=1.155$. A bin size of 5 was used for WHAM analysis, thus free energies are only available at potential energies in increments of 5 (black squares). The line is to guide the eye. (c) Fraction of folded states as a function of reduced temperature $(T)$.

$$
F_{\text {fold }}(T)=\frac{\sum_{V_{\text {nat }}<V} \Omega(V) \exp (-\beta V)}{\sum_{V} \Omega(V) \exp (-\beta V)} .
$$

At $T=1.156$, the populations of folded and unfolded states are equal. This alternative estimate of the folding transition temperature is in close agreement with that obtained from heat-capacity data. In previous studies concerning the same model protein, ${ }^{26}$ the authors monitor single-chain folding via the midpoint temperature, defined as the temperature where, on average, the protein maintains half $\left(N_{\text {mid }}=40.5\right)$ of the contacts that are present in its lowest energy state $\left(N_{\text {LES }}\right.$ $=81$ ). The midpoint temperature calculated in this study is 1.155 (data not shown), coinciding with estimates of the folding temperature from heat-capacity and melting-curve data. It is only slightly higher than that reported previously by Leonhard et al. $(T=1.146)^{26}$ and hence confirms the equivalence between the explicit solvent representation used in Ref. 26 and the implicit solvent representation employed in the present work. Further, the slight discrepancy is not surprising considering that the previous estimate was calculated using data only from individual simulations, while the calculations here benefited from the WHAM formalism.

Much experimental work has suggested that partially folded proteins are particularly susceptible to aggregation. ${ }^{33}$ Furthermore, a recent computational study has shown that aggregation rates are a strong function of folding cooperativity, with cooperative folders aggregating less rapidly. ${ }^{34}$ In order to determine whether our model protein populates partially folded states, or is a strict two-state folder, we have calculated its calorimetric cooperativity.

The experimental calorimetric criterion for two-state folding states that the van't Hoff enthalpy at the midpoint of the folding transition should be nearly equal to the calorimetric enthalpy of the entire phase transition. As our simulations are performed in the $N V T$ ensemble, we calculate energies instead of enthalpies. To meet the two-state criterion, the following quantity should be close to unity: ${ }^{35}$

$$
\kappa_{0}=\frac{\Delta U_{\mathrm{vH}}}{\Delta U_{\text {cal }}},
$$

where

$$
\Delta U_{\mathrm{vH}}=4 k_{B} T_{\text {mid }}^{2}\left(\frac{d \theta}{d T}\right)_{T=T_{\text {mid }}} .
$$

The quantity $\Delta U_{\mathrm{vH}}$ is the van't Hoff energy, which is calculated at the midpoint temperature $T_{\text {mid }}$. Here $T_{\text {mid }}$ corresponds to the temperature where the population of folded states $(\theta)$ is 0.5 , although other choices are possible. ${ }^{35}$ The quantity $\Delta U_{\text {cal }}$ corresponds to the calorimetric energy, which is the area under the heat capacity versus temperature curve, and in the present case $\Delta U_{\text {cal }}=110$. Using a $T_{\text {mid }}$ $=1.155, \Delta U_{\mathrm{vH}}=48$, resulting in a $\kappa_{0}$ of 0.43 . This is well below that for two-state folding proteins where experimentally measured $\kappa_{0}$ values are greater than 0.9 . However, experimental calculation of the calorimetric cooperativity often involves a baseline subtraction, where linear extrapolations of the native and denatured baselines are subtracted from $\Delta U_{\text {cal }}$, often leading to a much higher value of $\kappa_{0}$. Similar calculations have been performed for lattice-model proteins and have resulted in substantial increases in $\kappa_{0}{ }^{35}$ For the model system studied here, no native baseline could be subtracted, as at low temperatures, the heat capacity has significant-nonlinear character. However, a linear extrapolation was possible from high-temperature heat-capacity data, and was subtracted as in Refs. 35 and 36 . The resulting $\kappa_{0}$ showed a slight increase (from 0.43 to 0.47 ), but was far from meeting the calorimetric criterion for two-state folding. The single peak in the heat capacity suggests that no stable intermediate states exist. Therefore, the deviation from twostate behavior must be a consequence of conformational fluctuations in the folded and unfolded energy basins.

\section{B. Comparison of one-chain and two-chain thermodynamics}

Heat capacities for proteins are normally measured experimentally under dilute conditions, as irreversible aggrega- 
tion often occurs at high concentration. However, the nature of the systems studied here makes it possible to calculate the heat capacity for a pair of interacting proteins. Figure 4(a) shows the $C_{v}$ versus $T$ plots for one and two-chain systems. Given the box size $L=12$, the protein concentration in the latter system corresponds to the volume fraction $f_{v} \sim 7.5 \%$. A single peak is observed in both cases, indicating the presence of a single phase transition. However, the position of the peak $(T=1.12)$ occurs at a lower reduced temperature in the case of the isolated chain $(T=1.155)$.

To obtain further insight into this result, it is helpful to decompose the heat capacity from the two-chain system into its contributions from intra and interprotein interactions. The heat capacity is calculated via

$$
C_{v}=\frac{\left\langle V_{\mathrm{tot}}^{2}\right\rangle-\left\langle V_{\mathrm{tot}}\right\rangle^{2}}{k_{B} T^{2}},
$$

where $V_{\text {tot }}$ is the total potential energy, and the brackets denote thermodynamic averages. The quantity $V_{\text {tot }}$ can be expressed as the sum of contributions from intraprotein and interprotein interactions,

$$
V_{\text {tot }}=V_{\text {intra }}+V_{\text {inter }} \text {. }
$$

The heat capacity can be decomposed into its various contributions from intraprotein and interprotein energies:

$C_{v}=\frac{\left\langle V_{\text {intra }}^{2}\right\rangle-\left\langle V_{\text {intra }}\right\rangle^{2}+\left\langle V_{\text {inter }}^{2}\right\rangle-\left\langle V_{\text {inter }}\right\rangle^{2}+2^{*}\left(\left\langle V_{\text {intra }} V_{\text {inter }}\right\rangle-\left\langle V_{\text {intra }}\right\rangle\left\langle V_{\text {inter }}\right\rangle\right)}{k_{B} T^{2}}$.
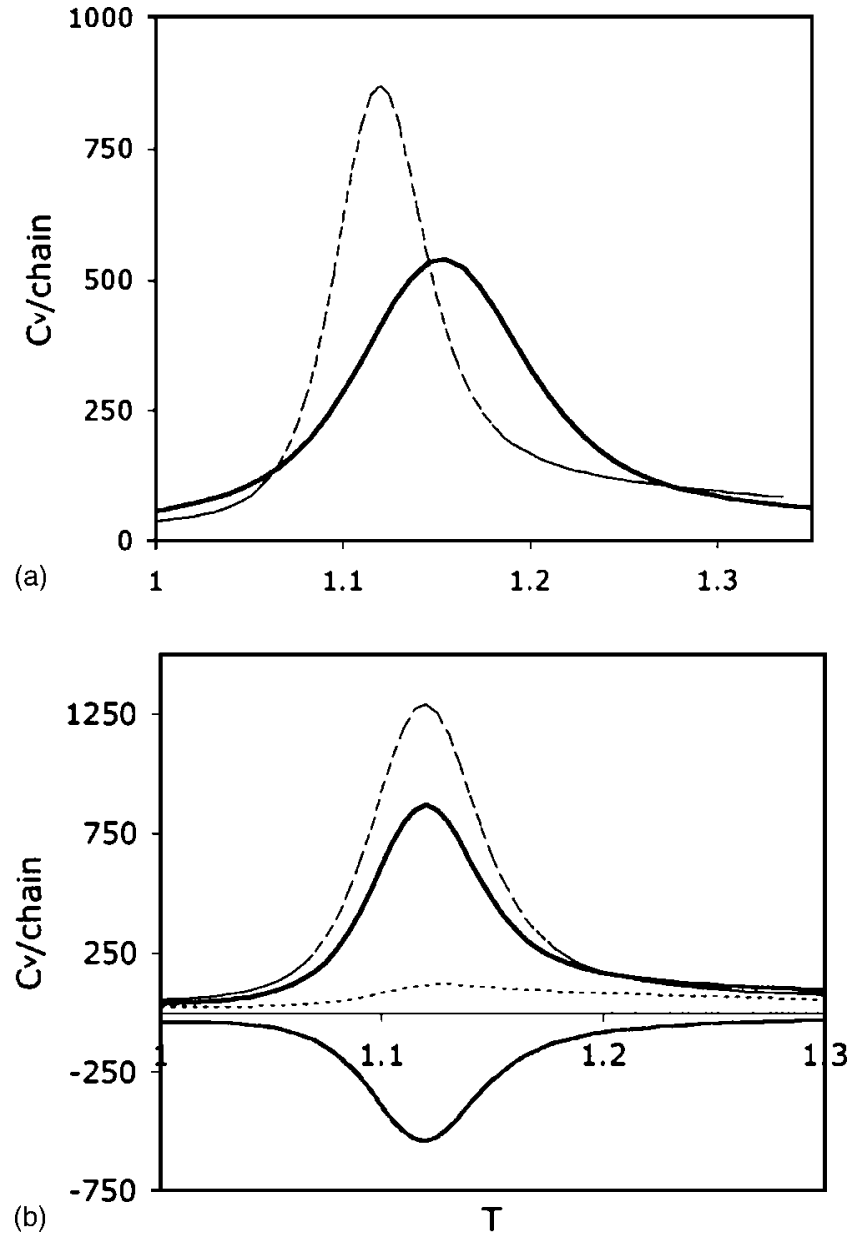

FIG. 4. (a) Heat capacity $\left(C_{v}\right)$ as a function of reduced temperature $(T)$ for one-chain (black line) and two-chain (dashed line) systems. (b) Heat capacity $\left(C_{v}\right.$, black line), fluctuations in intraprotein energy $\left(\delta_{\text {intra }}\right.$, large dashes), fluctuations in interprotein energy $\left(\delta_{\text {inter }}\right.$, small dashes), and correlation between intraprotein and interprotein energies $\left(\delta_{\mathrm{NC}}\right.$, grey line) as a function of reduced temperature $(T)$ for two-chain systems.
The contribution of fluctuations in intraprotein energy alone can be expressed via

$$
\delta_{\text {intra }}=\frac{\left\langle V_{\text {intra }}^{2}\right\rangle-\left\langle V_{\text {intra }}\right\rangle^{2}}{k_{B} T^{2}},
$$

and, correspondingly, the contribution from fluctuations in interprotein energy can be expressed as

$$
\delta_{\text {inter }}=\frac{\left\langle V_{\text {inter }}^{2}\right\rangle-\left\langle V_{\text {inter }}\right\rangle^{2}}{k_{B} T^{2}},
$$

the remaining contribution is

$$
\delta_{\mathrm{NC}}=\frac{2^{*}\left(\left\langle V_{\text {intra }} V_{\text {inter }}\right\rangle-\left\langle V_{\text {intra }}\right\rangle\left\langle V_{\text {inter }}\right\rangle\right)}{k_{B} T^{2}} .
$$

$\delta_{\mathrm{NC}}$ accounts for the (negative) correlation between $V_{\text {intra }}$ and

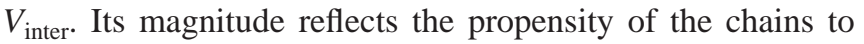
sacrifice intramolecular contacts for intermolecular contacts, and vice versa. $\delta_{\text {intra }}, \delta_{\text {inter }}$, and $\delta_{\mathrm{NC}}$, are plotted versus temperature in Fig. 4(b).

The quantity $\delta_{\text {intra }}$ shows a sharp peak at $T=1.12$ that is larger than that found for the net heat capacity. At $T$ $=1.12, \delta_{\text {intra }}$ is also much larger than $\delta_{\text {inter }}$, indicating that fluctuations in the intraprotein energy make a dominant contribution to the heat capacity. The negative value of the $\delta_{\mathrm{NC}}$ term indicates that intra and intermolecular energies do not fluctuate independently of each other as chains "trade" intramolecular energy for intermolecular energy. The magnitude of this quantity is also largest at $T=1.12$. The sum of these results shows that when in the presence of a second chain, individual molecules unfold at a lower temperature, and that unfolding is accompanied by a substantial degree of "trading" intramolecular energy for intermolecular energy. As the chains can move independently of each other, the 

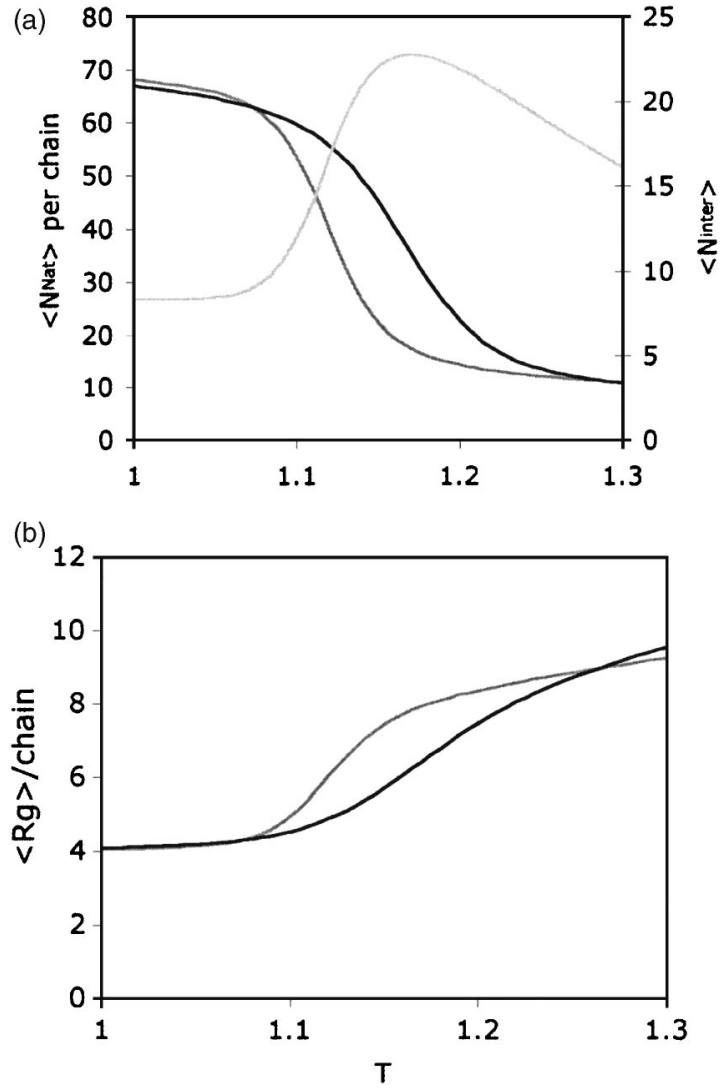

FIG. 5. (a) Average number of native $\left(\left\langle N_{\text {nat }}\right\rangle\right)$ contacts per chain for one(black line) and two-chain systems (dark grey line) $(T)$ and the average number of interprotein contacts $\left(\left\langle N_{\text {inter }}\right\rangle\right.$, light grey line $)$ as a function of reduced temperature. (b) Average radius of gyration $\left(R_{g}\right)$ as a function of reduced temperature $(T)$ for one- (black line) and two-chain (grey line) systems.

transition temperature also depends on the chain concentration, approaching the single-molecule value in highly diluted systems.

A direct verification of this conclusion is given in Fig. 5. The plots of average number of native contacts $\left(N_{\text {nat }}\right)$ versus temperature show that individual chains begin to unfold at lower temperatures when a second chain is present. At the folding temperature of isolated chains $(T=1.155)$, isolated chains on the average maintain 40 native contacts, whereas chains simulated in the presence of an additional chain only maintain 19 native contacts. The premature decrease in $\left\langle N_{\text {nat }}\right\rangle$ in the two-chain system is accompanied by an increase in the average number of interprotein contacts $\left(\left\langle N_{\text {inter }}\right\rangle\right)$ [Fig. 5(a)], as suggested by heat-capacity data. Last, average radius of gyration data shows that chains simulated in the presence of an additional chain begin to expand at lower temperatures than chains simulated in isolation [Fig. 5(b)].

\section{Free-energy surfaces for associating chains}

To gain further insight into the preferred micro-states of multichain systems, free-energy plots were generated as a function of $N_{\text {nat }}$ and $N_{\text {inter }}$ at various temperatures. The free energies plotted in Fig. 6 are relative free energies. Figure 6 displays the free energy surfaces for a two-chain system. Each of the first two plots $[T=1.0$ Fig. 6(a), $T=1.1$ Fig. 6(b) show a single minimum in free energy, corresponding to two chains in the native state with little mutual interaction. When the temperature is increased to 1.12 , the position of the peak in the heat capacity [Fig. 4(a)], a second minimum appears, corresponding to chains that have sacrificed a considerable amount of native structure for an increase in interprotein contacts. At temperatures greater than the folding temperature of an individual chain in isolation $(T=1.155)$, only the free energy minimum at low $N_{\text {nat }}$ survives showing that the system essentially consists of only unfolded chains with some degree of association, which, on average [Fig. 4(a)], decreases with further increases in temperature.

At $T=1.12$, chains in isolation populate the folded state $80 \%$ of the time [Fig. 3(c)]. As shown in Fig. 6(c), in a two-chain system at this temperature, chains remain folded only about $50 \%$ of the time. To understand better the driving forces for this change in conformational preferences, the two minima in Fig. 6(c) were investigated more closely. The minimum with a large $N_{\text {nat }}$ corresponds to native-like dimers, while the second minimum of roughly the same free energy corresponds to a non-native aggregate. The smaller number of total contacts in systems comprising the second minimum suggests that it is entropically favored. As shown in Fig. 7, the non-native aggregates populate states with much higher values of potential energy than the native-like dimer, confirming this hypothesis.

An attempt was made to locate transition-state structures separating these two states. However, there is a great deal of configurational space separating the two minima, resulting in a variety of possible low energy pathways for the system to traverse in traveling from one state to another. Thus it proved difficult to identify well-defined saddle regions that may represent transition states. It is possible that adding additional coordinates (radius of gyration, system potential energy, etc.) may assist in elucidating transition state structures.

\section{Contact maps for two-chain systems}

Contact maps (Fig. 8) were generated to characterize further the two minima in Fig. 6(c). The darkness of the box pertaining to each contact is representative of that particular contact's probability of occurrence. As there are two chains, the intraprotein probabilities have been normalized to reflect the fact that each contact may occur twice for any given snapshot. For example, if the contact 54-57 is present for each chain, during each snapshot, then its probability is unity. Interprotein contact probabilities have not been normalized in this fashion, and thus reflect the probability that a given contact is present.

Figure 8(a) shows data pertaining to native dimers that have 5-10 intermolecular contacts and 120-140 intramolecular contacts. Snapshots of a native-like dimer are shown in Fig. 9(a). Data in panel 8(b) pertains to non-native aggregates that consist of chains having 20-30 intermolecular contacts, and 25-35 intramolecular contacts. Snapshots of this state are shown in Fig. 9(b). Each contact map is divided into two parts. The probabilities for intraprotein contacts and interprotein contacts are represented below and above the diagonal, respectively. 
(a)

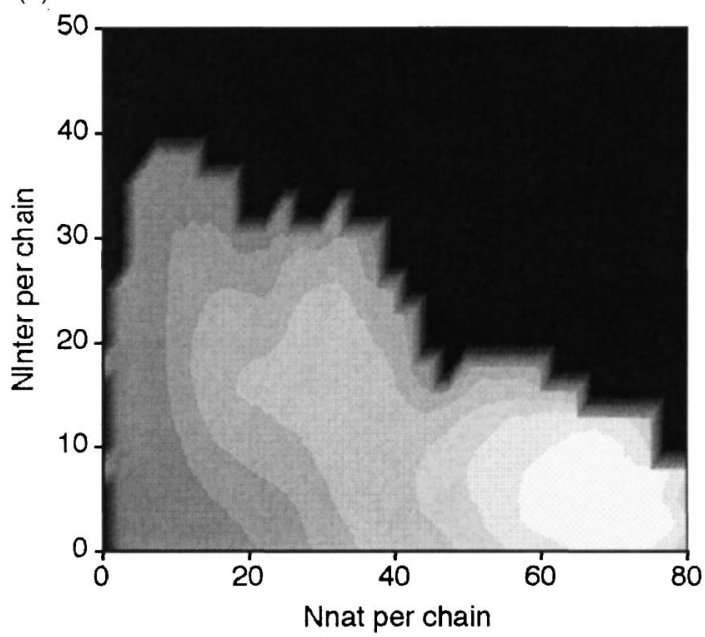

(c)

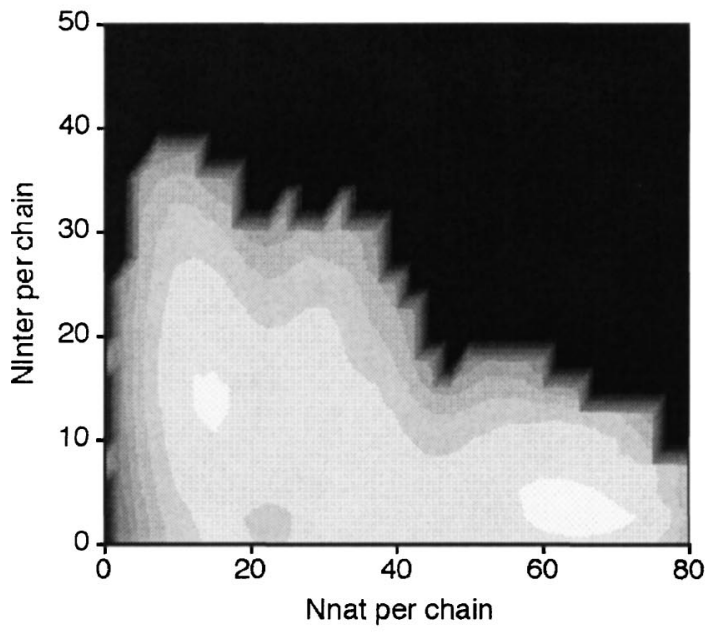

(b)

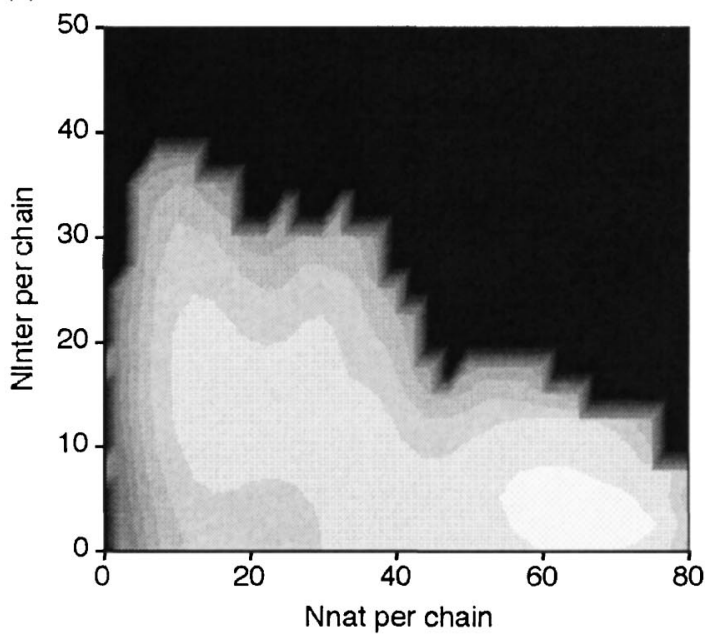

(d)

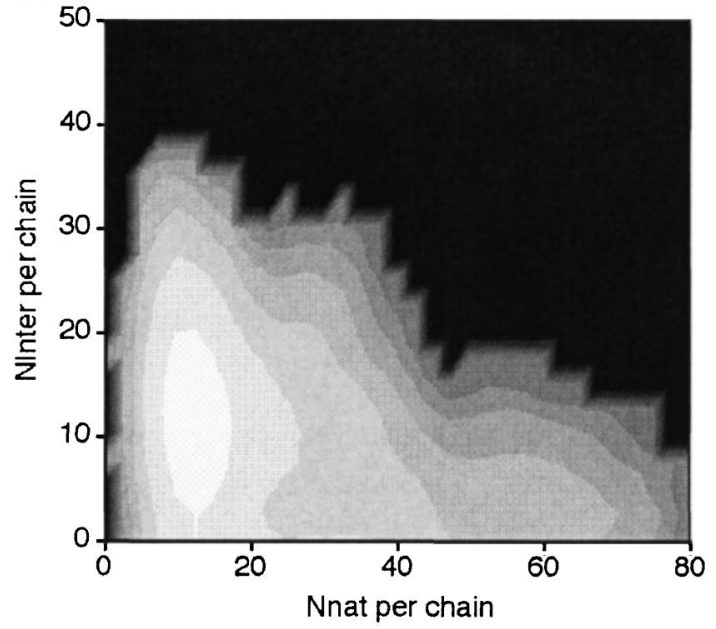

(e)

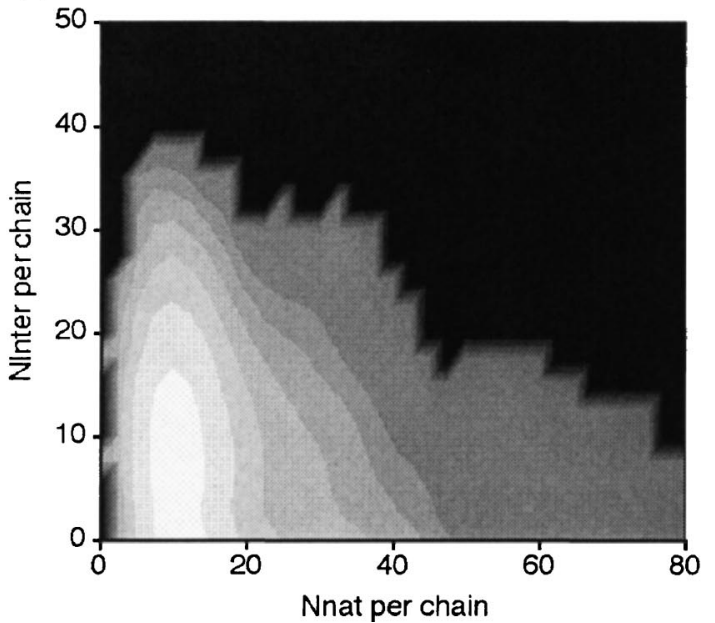

FIG. 6. Free energy (contours) vs the number of native $\left(N_{\text {nat }}\right)$ and interprotein contacts $\left(N_{\text {inter }}\right)$ for two-chain simulations. (a) $T=1.0$, (b) $T=1.1$, (c) $T$ $=1.12$, (d) $T=1.2$, (e) $T=1.3$. Contours are in increments of 5, decreasing in value from black (75-80) to white (0-5).

As shown in Fig. 8(a), only a few specific intermolecular contacts persist in the native-like dimer state. They occur between the segment of beads 1-3 (residue types $K E K$ ) on one chain, and the segments 24-37-46 (DKD) or 26-35-48 $(D K E)$ on the second chain. These contacts occur as a consequence of charge patterns. The segment of beads, $1-3$, has a pattern of,,+-+ , while the two segments $24-37-46$, and 26-35-48 have patterns of,,-+- . Coulombic interactions between ionic residues are implicitly included in MJ interresidue potentials. The proper alignment of these sets of amino acids can result in three favorable interprotein contacts, with energies of -6 and $-6.5 k_{B} T_{0}$, respectively. Also, 


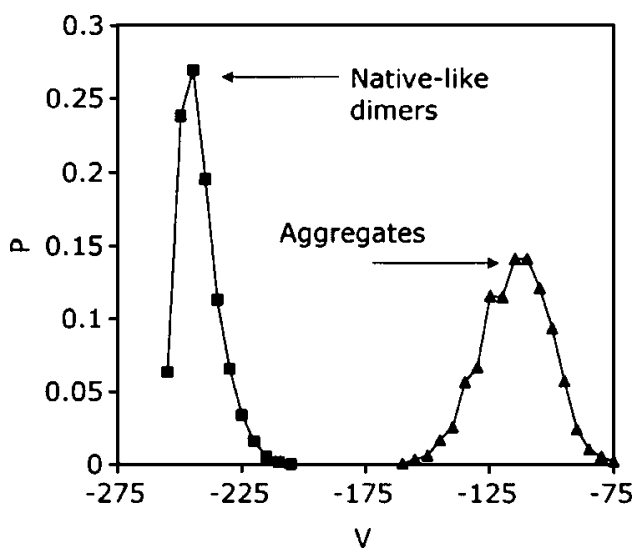

FIG. 7. Probability $(P)$ of a two-chain system with a potential energy $(V)$. Native-like dimers consist of two-chain systems that have 120-140 intraprotein contacts and 5-10 interprotein contacts, while non-native aggregates consist of two-chain systems with 25-35 intraprotein contacts and 20-30 interprotein contacts at $T=1.12$.

note that the pair of segments, 24-37-46 and 26-35-48, consist of beads that are adjacent in the native state, but not in primary sequence. The interactions of these beads with beads 1-3 on a separate chain provide an example of how native state topology can dictate interprotein interactions.

It is also interesting to compare the intraprotein contact map from the native-like dimers with that generated for the lowest-energy structure. Intraprotein contact probabilities are located below that diagonal in Fig. 8(a). For the most part, they are quantitatively the same; 61 of the 81 native contacts (75\%) are present at least $70 \%$ of the time. However there is noticeable deviation in the lower left-hand portion of the plot. A closer look at the results shows that there are eight contacts present in the LES that are populated less than 50\% of the time in the native-like dimer state. All of these contacts involve beads either at the corners of the cubic LES, or one bead removed from the corners. This result is easy to rationalize in terms of the topology of the LES, as beads at the corners can only form one nonlocal contact, whereas beads one lattice position from the corner can only form two nonlocal contacts. Last, as expected, non-native contacts contribute little (less than $2 \%$ on average) to the overall system potential energy when native-like dimers are present.

The contact map for the non-native aggregates is significantly different from that for the native-like dimers. The increase in interprotein association is evident when comparing the area above the diagonal for plot $8(\mathrm{~b})$ with that in $8(\mathrm{a})$. Further, the higher entropy of the aggregated state is manifested in the broad distribution of observed contacts. Only two interprotein contacts exist greater than $50 \%$ of the time, nine exist at least $20 \%$ time, while 41 exist in greater than $10 \%$ of the snapshots sampled. Nearly half (20) of these 41 contacts occur in the LES for a single chain, showing that a domain-swapping-like mechanism is involved in aggregation. The ten most probable contacts are listed in Table I, the first eight of which involve at least one bead buried in the LES. This result suggests that exposure of the protein core is required for the formation of a non-native aggregate.

Some evidence of native-like structure remains for the non-native aggregates, but relatively few of the native con-

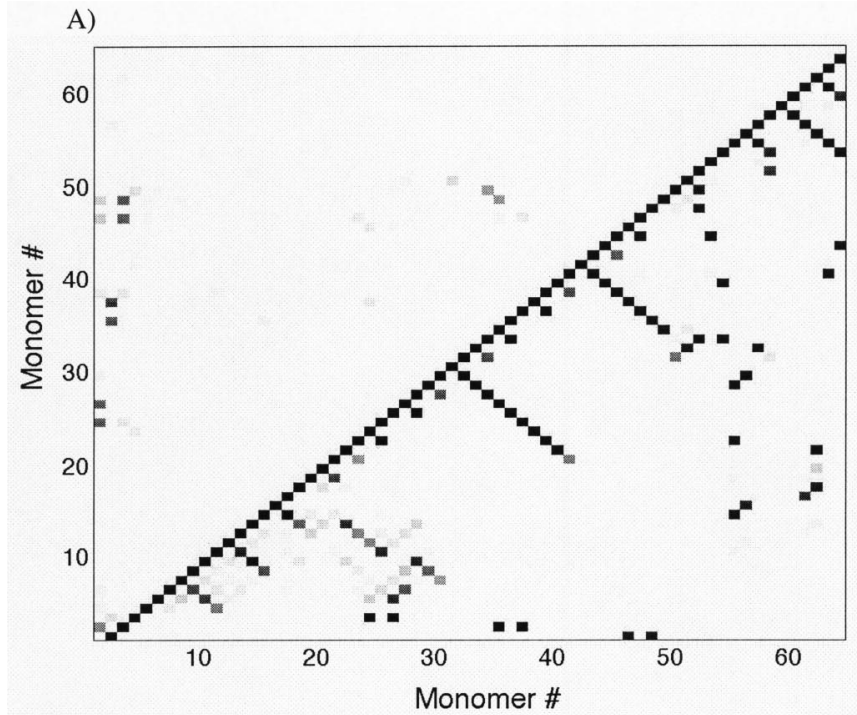

B)

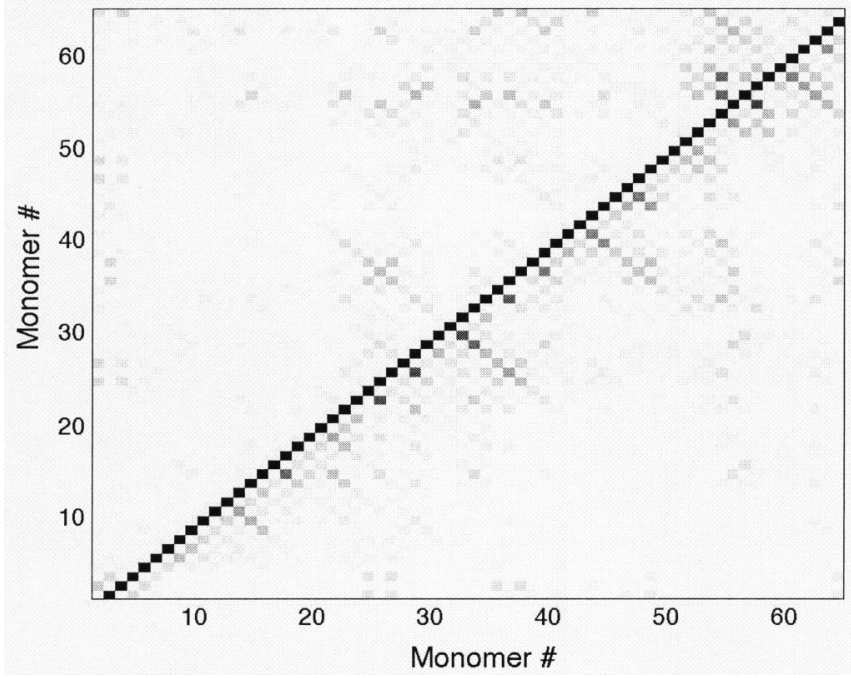

FIG. 8. Contact maps for a two-chain system. The axes are each numbered 1-64, consistent with the number of beads in each chain. The area above the diagonal corresponds only to interprotein contacts, while the area below only pertains to intraprotein contacts. Boxes represent the probability of occurrence for each contact. Black corresponds to a probability of 1 , and white to a probability of 0 . Increments are in units of 0.05 . (a) Only includes data from states with 120-140 total intraprotein contacts and 5-10 interprotein contacts. (b) Only includes data from states with 25-35 total intraprotein contacts and 20-30 interprotein contacts.

tacts persist in the aggregated state. Figure 10 shows the number of intraprotein contacts observed with a specified probability, and the ten most probable contacts are listed in Table II. Note that with the exception of the contact 28-33, all the contacts listed in Table II involve beads separated by two beads in primary sequence; the minimum distance for nonbonded contacts. This result shows that when aggregated, individual proteins have lost most of their nonlocal structure, but tend to maintain some local contacts. This is not surprising, as in a two-chain system individual molecules are significantly expanded at $T=1.12$ [Fig. 5(b)]. Furthermore, five of the ten contacts in Table II involve pairs of beads buried in the native state, showing that some of the native-state core tends to persist when a chain is aggregated. Additionally, while all of the contacts listed in Table II are native contacts, 

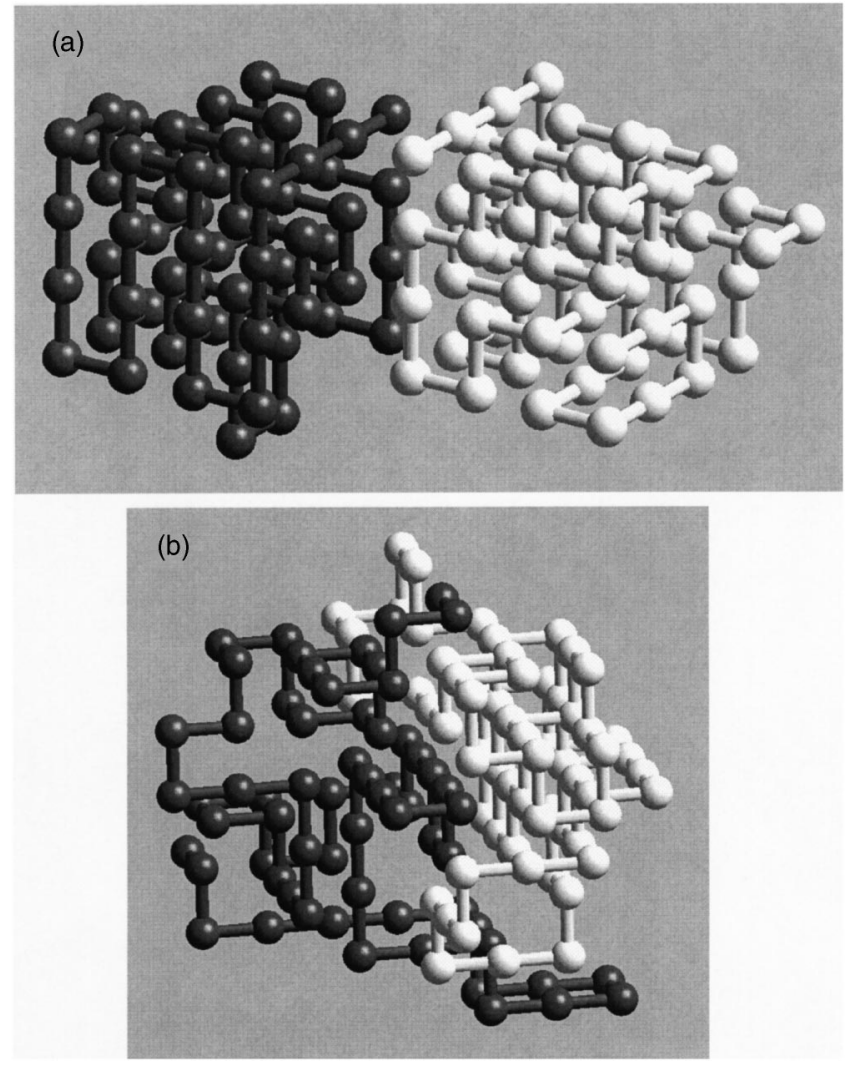

FIG. 9. (a) Snapshot of a two-chain system (native-dimer) with a total of 144 intramolecular contacts, and seven intermolecular contacts. (b) Snapshot of a two-chain system (non-native aggregate) with a total of 35 intramolecular contacts, and 26 intermolecular contacts.

non-native contacts do substantially stabilize the aggregated state. On average, they contribute $25 \pm 6 \%$ of the total potential energy.

\section{CONCLUSIONS}

Computer simulations of coarse-grained model proteins have contributed significantly to our understanding of the protein-folding problem. A logical extension of this work is the investigation of systems of proteins, where interprotein interactions compete with intraprotein interactions and thus affect the partitioning of folded and unfolded states. This phenomenon is of much practical interest, as protein aggre-

TABLE I. Ten most probable interprotein contacts observed within nonnative aggregates. An asterisk $(*)$ denotes that the bead is buried in the lowest-energy structure. Energies are expressed in the units $k_{B} T_{0}$.

\begin{tabular}{ccc}
\hline \hline Contact & Probability & Energy \\
\hline $54 \mathrm{M}^{*}-55 \mathrm{I}^{*}$ & 0.52 & -2.982 \\
$54 \mathrm{M}^{*}-57 \mathrm{~W}$ & 0.51 & -3.942 \\
$33 \mathrm{I}^{*}-54 \mathrm{M}^{*}$ & 0.25 & -2.982 \\
$28 \mathrm{~L}^{*}-55 \mathrm{I}^{*}$ & 0.23 & -2.843 \\
$55 \mathrm{I}^{*}-62 \mathrm{~L}$ & 0.21 & -2.843 \\
$36 \mathrm{~L}^{*}-55 \mathrm{I}^{*}$ & 0.20 & -2.843 \\
$54 \mathrm{M}^{*}-63 \mathrm{P}$ & 0.20 & -2.438 \\
$25 \mathrm{I}^{*}-36 \mathrm{~L}^{*}$ & 0.20 & -2.843 \\
$37 \mathrm{~K}-64 \mathrm{E}$ & 0.20 & -2.347 \\
$35 \mathrm{~K}-64 \mathrm{E}$ & 0.18 & -2.347 \\
\hline \hline
\end{tabular}

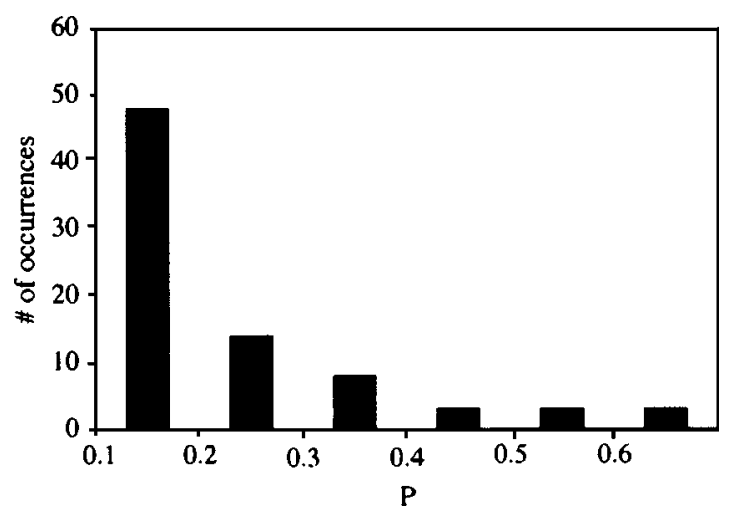

FIG. 10. Number of intraprotein contacts with a specified probability $(P)$ for non-native aggregates.

gation has been implicated in nearly 20 human diseases and creates a serious problem during the manufacture of pharmaceutical proteins.

In the current study, we have focused on studying a system of two protein chains; the simplest possible system to investigate the effects of interprotein interactions on single molecule conformational preferences. At the lowest temperature studied, chains prefer their native states, but do associate weakly through sets of beads on the protein surface. As the temperature is raised, individual molecules tend to populate unfolded states more often when a second chain is present. In part, this is a consequence of a trade between intramolecular energy and intermolecular energy. Contact maps provide a direct explanation for this phenomenon, as a majority of the most probable interprotein contacts involve beads that are buried in the protein core in the native state. Conformational fluctuations at higher temperatures make these beads available for interactions between proteins, and thus facilitate aggregation. Numerous experimental studies have indeed shown that aggregation tends to be favored when proteins are destabilized. Last, non-native, intraprotein contacts made a significant contribution to aggregate stability. To our knowledge, this is the first time such a phenomenon has been observed in experimental or computational studies.

Entropy is a driving force for association under partially denaturing conditions. We are unaware of any experimental data that can directly verify this result. It does not seem

TABLE II. Ten most probable intraprotein contacts observed within nonnative aggregates. An asterisk $(*)$ denotes that the bead is buried in the lowest-energy structure. Energies are expressed in the units $k_{B} T_{0}$.

\begin{tabular}{ccc}
\hline \hline Contact & Probability & Energy \\
\hline $54 \mathrm{M}^{*}-57 \mathrm{~W}$ & 0.64 & -3.942 \\
$25 \mathrm{I}^{*}-28 \mathrm{~L}^{*}$ & 0.60 & -2.843 \\
$29 \mathrm{Q}-32 \mathrm{P}$ & 0.60 & -1.606 \\
$22 \mathrm{~L}^{*}-25 \mathrm{I}^{*}$ & 0.59 & -2.843 \\
$33 \mathrm{I}^{*}-36 \mathrm{~L}^{*}$ & 0.56 & -2.843 \\
$28 \mathrm{~L}^{*}-33 \mathrm{I}^{*}$ & 0.52 & -2.843 \\
$14 \mathrm{~L}-17 \mathrm{~V}$ & 0.49 & -2.102 \\
$36 \mathrm{~L}^{*}-39 \mathrm{~F}^{*}$ & 0.44 & -2.411 \\
$44 \mathrm{~F}-47 \mathrm{~V}$ & 0.41 & -1.958 \\
$57 \mathrm{~W}-60 \mathrm{Y}$ & 0.39 & -1.472 \\
\hline
\end{tabular}


plausible that protein self-association into large assemblies such as inclusion bodies and amyloid fibrils results in increased protein entropy. It is possible that our result is limited to small systems, as chains in larger aggregates will lose configurational entropy upon interactions with more than one partner. Further, we consider main-chain interactions, including hydrogen bonds, only implicitly. Such interactions are of great importance in amyloid fibril formation, but are unavoidably modulated by specific side chain effects. Simulations of larger systems are being launched in an effort to further understand the above-presented result.

Despite the simplicity of the system studied here, the simulations have been able to capture some key aspects of protein aggregation phenomena observed experimentally. Further, the simplicity of the system has also facilitated a thermodynamic characterization that has yielded several novel results. Future studies will be directed toward studying larger and more complex systems, as well as mutagenesis studies aimed at further uncovering the generic aspects of protein aggregation.

\section{ACKNOWLEDGMENTS}

This work is supported by the National Science Foundation under Grant No. BES-0118208 and by the Office of Basic Sciences U.S. Department of Energy. The authors gratefully acknowledge assistance from Adam Meadows in preparing the contact maps.

${ }^{1}$ C. Dobson, Philos. Trans. R. Soc. London, Ser. B 356, 133 (2001).

${ }^{2}$ K. A. Dill and H. S. Chan, Nat. Struct. Biol. 4, 10 (1997).

${ }^{3}$ A. R. Dinner, A. Sali, L. J. Smith, C. M. Dobson, and M. Karplus, Trends Biochem. Sci. 25, 331 (2000).

${ }^{4}$ J. W. Kelly, Curr. Opin. Struct. Biol. 8, 101 (1998).

${ }^{5}$ E. D. Clark, Curr. Opin. Biotechnol. 12, 202 (2001).

${ }^{6}$ H. Lilie, E. Schwarz, and R. Rudolph, Curr. Opin. Biotechnol. 9, 497 (1998).
${ }^{7}$ S. Misawa and I. Kumagai, Biopolymers 51, 297 (1999).

${ }^{8}$ E. Y. Chi, S. Krishnan, T. W. Randolph, and J. F. Carpenter, Pharmacol. Res. 20, 1325 (2003).

${ }^{9}$ D. Bratko and H. W. Blanch, J. Chem. Phys. 114, 561 (2001).

${ }^{10}$ D. Bratko and H. W. Blanch, J. Chem. Phys. 118, 5185 (2003).

${ }^{11}$ G. Giugliarelli, C. Micheletti, J. M. Banavar, and A. Maritan, J. Chem. Phys. 113, 5072 (2000)

${ }^{12}$ R. I. Dima and D. Thirumalai, Protein Sci. 11, 1036 (2002).

${ }^{13}$ P. Gupta, C. K. Hall, and A. Voegler, Fluid Phase Equilib. 160, 87 (1999).

${ }^{14}$ S. Istrail, R. Schwartz, and J. King, J. Comput. Biol. 6, 143 (1999).

${ }^{15}$ L. Toma and S. Toma, Biomacromolecules 1, 232 (2000).

${ }^{16}$ H. D. Nguyen and C. K. Hall, Proc. Natl. Acad. Sci. U.S.A. 101, 16180 (2004).

${ }^{17}$ T. Cellmer, D. Bratko, J. M. Prausnitz, and H. Blanch, Biotechnol. Bioeng. 89, 78 (2005).

${ }^{18}$ H. D. Nguyen and C. K. Hall, Biotechnol. Bioeng. 80, 823 (2002).

${ }^{19}$ P. M. Harrison, H. S. Chan, S. B. Prusiner, and F. E. Cohen, J. Mol. Biol. 286, 593 (1999).

${ }^{20}$ P. M. Harrison, H. S. Chan, S. B. Prusiner, and F. E. Cohen, Protein Sci. 10, 819 (2001).

${ }^{21}$ S. Miyazawa and R. L. Jernigan, Macromolecules 18, 534 (1985).

${ }^{22}$ K. Leonhard, J. M. Prausnitz, and C. J. Radke, Protein Sci. 13, 358 (2004).

${ }^{23}$ S. A. Hassan, J. Phys. Chem. B 108, 19501 (2004).

${ }^{24}$ S. Kumar, D. Bouzida, R. Swendsen, P. A. Kollman, and R. J. A., J. Comput. Chem. 13, 1011 (1992).

${ }^{25} \mathrm{H}$. Li, C. Tang, and N. S. Wingreen, Phys. Rev. Lett. 79, 765 (1997).

${ }^{26}$ K. Leonhard, J. M. Prausnitz, and C. J. Radke, Phys. Chem. Chem. Phys. 5, 5291 (2003).

${ }^{27}$ K. Leonhard, J. M. Prausnitz, and C. J. Radke, Biophys. Chem. 106, 81 (2003).

${ }^{28}$ D. Frenkel and B. Smit, Understanding Molecular Simulation (Academic, New York, 1996).

${ }^{29}$ A. Sali, E. Shakhnovich, and M. Karplus, Nature (London) 369, 248 (1994).

${ }^{30}$ F. T. Wall and F. Mandel, J. Chem. Phys. 63, 4592 (1975).

${ }^{31}$ D. Gront, A. Kolinski, and J. Skolnick, J. Chem. Phys. 113, 5065 (2000).

${ }^{32}$ M. Cecchini, F. Rao, M. Seeber, and A. Caflisch, J. Chem. Phys. 121, 10748 (2004).

${ }^{33}$ A. L. Fink, Folding Des. 3, R9 (1998).

${ }^{34}$ L. Clark, Protein Sci. 14, 653 (2005).

${ }^{35}$ H. Kaya and H. S. Chan, Proteins 40, 637 (2000).

${ }^{36}$ Y. Zhou, C. K. Hall, and M. Karplus, Protein Sci. 8, 1064 (1999). 